\title{
The Risk of Gout in Patients with Psoriasis: A Population-Based Cohort Study in Taiwan
}

\author{
James Cheng-Chung Wei ${ }^{1-3}$, Yi-Jung Chang $\mathbb{D}^{4}$, Yu-Hsun Wang ${ }^{5}$, Chih-Jung Yeh $\mathbb{D}^{6}$ \\ 'Department of Allergy, Immunology \& Rheumatology, Chung Shan Medical University Hospital, Taichung, Taiwan; ${ }^{2}$ Institute of Medicine, College of \\ Medicine, Chung Shan Medical University, Taichung, Taiwan; ${ }^{3}$ Graduate Institute of Integrated Medicine, China Medical University, Taichung, Taiwan; \\ ${ }^{4}$ Department of Pediatrics, Chang Gung Memorial Hospital, Chang Gung University College of Medicine, Taoyuan, Taiwan; ${ }^{5}$ Department of Medical \\ Research, Chung Shan Medical University Hospital, Taichung, Taiwan; ' ${ }^{6}$ epartment of Public Health, Chung Shan Medical University, Taichung, Taiwan
}

Correspondence: Chih-Jung Yeh, Department of Public Health, Chung Shan Medical University, No. II0, Sec. I, Jianguo N. Road, South District, Taichung, 4020I, Taiwan, Tel +886 424739595 \#I 1794, Fax +886 4 24637389, Email alexyeh@csmu.edu.tw

Background: Previous research has pointed to the relationship between psoriasis and the development of gout. However, most previous studies had either small sample sizes or short study durations. Therefore, in this nationwide cohort study, we investigated the effect of psoriasis on the risk of gout development.

Methods: The study group included one million patients from Taiwan, whom we followed for 14 years. The participants were divided into two cohorts designated as psoriasis and non-psoriasis. A 1:4 propensity score matching test was used to compare age, sex, and index year between the two cohorts. Cox proportional hazard regression was used to determine the hazard ratios (HRs) and $95 \%$ confidence intervals (CIs) for the risk of gout. Sensitivity analyses were conducted to evaluate the HR for gout after the occurrence of psoriasis.

Results: The incidence densities of gout in the psoriasis and non-psoriasis cohorts were 6.96 and 5.09 per 1000 person-years, respectively. After adjusting for age, sex, urbanization, comorbidities, and nonsteroidal anti-inflammatory drug (NSAID) use, the adjusted hazard ratio (aHR) with 95\% CI for incidental gout in the psoriasis group was 1.38 (1.2-1.6). Moreover, the aHR (95\% CI) values for gout risk in patients with psoriasis using NSAIDs and those who did not were 1.21 (1.0-1.47) and 1.65 (1.33-2.05), respectively.

Conclusion: This study demonstrated an association between psoriasis and risk of developing gout. Clinically, patients with psoriasis should be evaluated for incidental gout.

Keywords: psoriasis, gout, epidemiology, corticosteroids, NHIRD, cohort

\section{Introduction}

Psoriasis is a chronic inflammatory skin disease affecting people worldwide, with a prevalence of approximately $2 \%$. ${ }^{1}$ It typically affects the skin and is characterized by scaly, erythematous plaques that are highly visible and can lead to stigmatization. Furthermore, it may considerably affect the patient's quality of life and psychosocial function. ${ }^{2}$ Furthermore, the medical cost associated with psoriasis is high. ${ }^{3,4}$ In 2014, the World Health Organization recognized psoriasis as a serious non-communicable disease and in an accompanying report, emphasized the need to better understand the global burden of this disease. ${ }^{5}$ Recent studies have revealed that it is not only a dermatological disease but is also associated with several systemic inflammatory disorders, thus reducing a patient's lifespan. ${ }^{6-8}$

Gout is the most common inflammatory arthritis that causes recurrent episodes of joint pain and swelling, ${ }^{9}$ and its prevalence has increased globally in recent years, thus increasing the burden of the disease. Gout is characterized by a rise in blood uric acid level with age. The long-term goal of therapy is to prevent recurrent gout flares. This is achieved through a combination of strategies for risk reduction, in addition to diet control and urate-lowering drug therapy. ${ }^{10}$ However, patients with gout are likely to experience comorbidities ${ }^{11,12}$ that are associated with poor outcomes and the need for complex clinical management. 
Substantial progress has been made in elucidating comorbidities associated with psoriasis. However, only a few largescale studies have been conducted in this field. While the coexistence of psoriasis and gout has been documented, ${ }^{13-16}$ the association between them is not clearly defined. Therefore, we investigated this association and explored the risk factors for developing gout using data from Taiwan's National Health Insurance Research Database (NHIRD).

\section{Methods}

\section{Data Source}

Data from the National Institutes of Health in Taiwan were used for this cohort study. Since 1995, Taiwan's National Health Insurance Plan has provided universal medical insurance to the country's population (approximately 23 million to date). Enrolment in this government-funded health insurance system is mandatory, and the coverage rate exceeds $98 \%$ of the Taiwanese population. The NHIRD contains information from this health insurance system, including patient demographic data, inpatient and outpatient claims, and diagnostic codes. This research database has also been validated in many studies. ${ }^{17-19}$ Since the data in the NHIRD are encrypted and de-identified, patient confidentiality is protected. This study was approved by the Institutional Review Board of the Chung Shan Medical University Hospital.

\section{Study Population}

We randomly selected one million patients between January 2000 and December 2013 from the NHIRD (Figure 1). Psoriasis was identified based on the presence of at least three outpatient claims or one inpatient claim according to the International Classification of Diseases, Ninth Revision, Clinical Modification (ICD-9-CM) code 696.8 to ensure the validity of the diagnosis. The cohort entry date was defined as the date of the psoriasis diagnosis. We excluded people with ICD-9-CM code 696.0 for psoriatic arthropathy and those with gout diagnosed before the entry year. Patients diagnosed with psoriasis between 2000 and 2012 were included in the psoriasis cohort. Owing to the consistent index

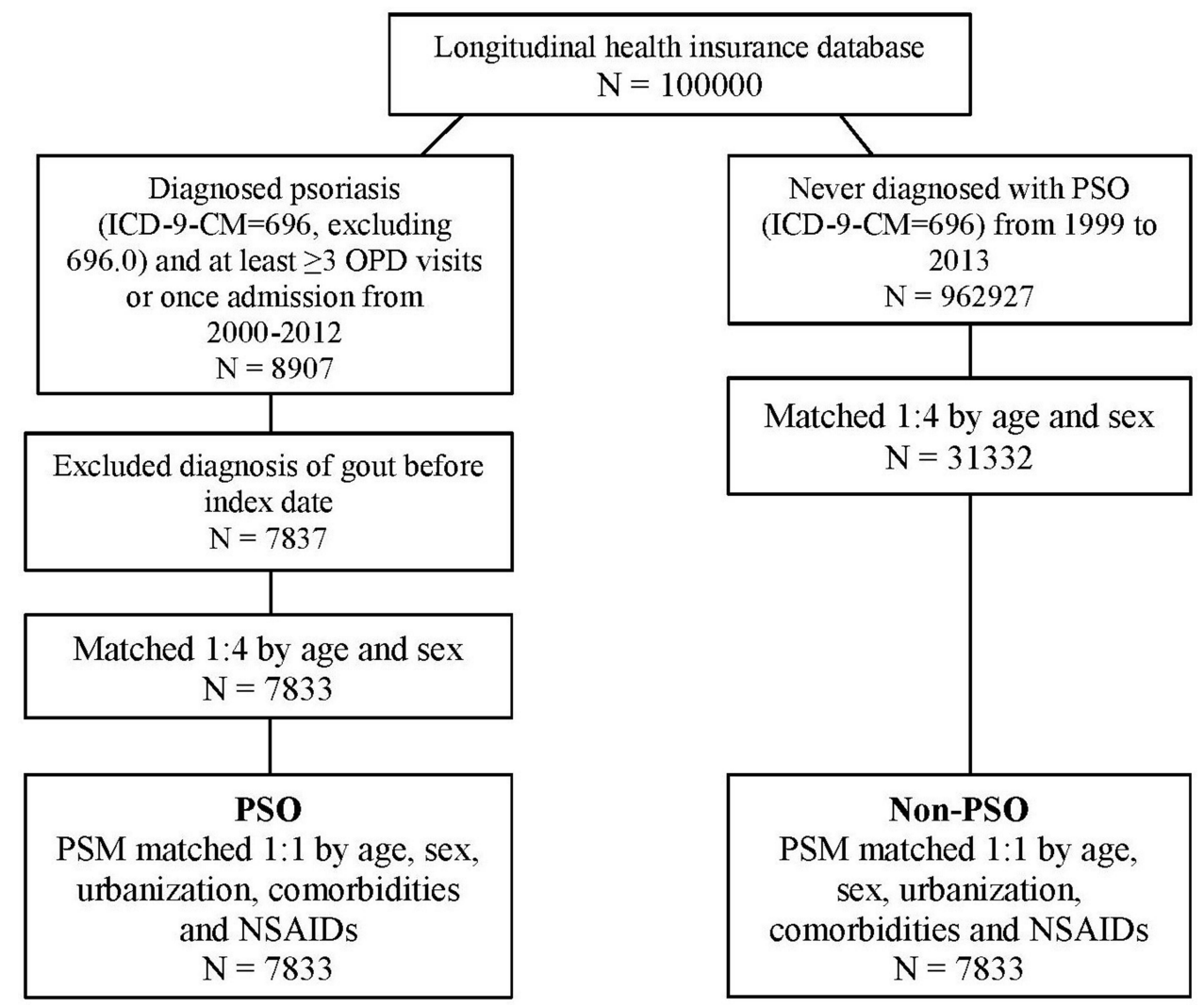

Figure I Flow chart of the study design.

Abbreviation: PSO, psoriasis. 
date, the non-psoriasis cohort included patients without psoriasis who were individually matched in a 1:4 ratio to the participants in the psoriasis cohort based on age, sex, and index year. Another non-psoriasis cohort included patients without psoriasis who were matched with a 1:1 propensity score to the participants in the psoriasis cohort. The variables used in propensity score matching were age, sex, urbanization, comorbidities, and nonsteroidal anti-inflammatory drug (NSAID) use. The propensity score was estimated using logistic regression, and binary variables were the psoriasis and non-psoriasis groups. Propensity score matching helped minimize demographic differences between the two cohorts. ${ }^{20}$

\section{Main Outcome and Comorbidities}

The main outcome of this study was the incidence of gout in the psoriasis and non-psoriasis cohorts. Gout was defined as the presence of at least three outpatient claims or one inpatient claim according to the ICD-9-CM code 274. Both groups were followed until the onset of gout, withdrawal from the national insurance system, or December 31, 2013, whichever occurred first. Since the association between gout and psoriasis may be confounded by multiple concurrent diseases, we identified the following comorbidities based on their ICD-9-CM diagnostic codes: hypertension (401-405), hyperlipidemia (272.0-272.4), diabetes mellitus (250.x), obesity (278.0), chronic liver disease (571), chronic renal disease (585), chronic obstructive pulmonary disease ([COPD] 491, 492, 496), and autoimmune disease (710.x, 714.x, 720.x). Diffuse diseases of the connective tissue (710.x), rheumatoid arthritis and other inflammatory polyarthropathies (714.x), and ankylosing spondylitis and other inflammatory spondylopathies (720.x) were included as autoimmune diseases. These comorbidities were considered if they were diagnosed one year before the entry date. Additionally, NSAID usage was considered when they had been used for $\geq 30$ days.

\section{Statistical Analysis}

Absolute standardized difference (ASD) was used to evaluate the balance in the baseline characteristics of the age-, sex-, and propensity score-matched populations. An ASD $<0.1$ was not considered a statistically significant difference. We used the Kaplan-Meier method to obtain the cumulative incidence curve and the Log rank test to evaluate the differences between the psoriasis and non-psoriasis cohorts. Univariate and multivariate Cox proportional hazard regression were used to determine hazard ratios (HRs), and 95\% confidence intervals (CIs) were used to determine the association between gout, psoriasis, and other risk factors. Additionally, we performed Cox proportional hazard regression after stratification by age, sex, urbanization, and comorbidities at the start of the follow-up period. To assess the robustness of our findings, we conducted a sensitivity analysis to further examine the association between a history of psoriasis, no psoriasis, and gout risk. In the sensitivity analysis, the diagnosis codes of psoriasis treated with methotrexate, cyclosporin, and allopurinol while those of gout treated with benzbromarone, sulfinpyrazone, and probenecid were considered. In this model, the diagnoses of psoriasis and gout were restricted to those made by subspecialists, including dermatologists and rheumatologists. The sensitivity analysis was performed to evaluate the risk of gout after the occurrence of psoriasis. All statistical analyses were performed using the SAS software, version 9.4 (SAS Institute, Cary, NC, USA).

\section{Results}

In this study, 7833 people with psoriasis were included and matched to 7833 people without psoriasis. The baseline characteristics of the patients are summarized in Table 1. The mean age of patients in the psoriasis and non-psoriasis cohorts was similar $(37.8 \pm 20.1$ vs $37.9 \pm 20.3$ years). The psoriasis cohort consisted of $4076(52.0 \%)$ men and 3757 $(48.0 \%)$ women, whereas the non-psoriasis cohort consisted of $4059(51.8 \%)$ men and $3774(48.2 \%)$ women. Hypertension was the most common comorbidity among the patients with psoriasis. Up to two of the patients used NSAIDs.

We also analyzed the incidence density of gout in the different groups (Table 2). The incidence densities of gout in the psoriasis and non-psoriasis cohorts were 6.96 and 5.09 per 1000 person-years, respectively. A primary composite outcome event (gout) occurred in $433(5.5 \%)$ and 329 patients $(4.2 \%)$ in the psoriasis and non-psoriasis groups, respectively (6.9 vs 5.0 events per 1000 patient-years; HR, 1.37; 95\% CI, 1.19-1.58). The Kaplan-Meier analysis showed that by the end of the 14-year follow-up period, the psoriasis cohort exhibited a higher cumulative incidence of gout than in the non-psoriasis cohort (Supplementary Figure S1). 
Table I Demographic and Clinical Characteristics of Study Population

\begin{tabular}{|c|c|c|c|c|c|c|}
\hline & \multicolumn{2}{|c|}{ Before Propensity Score Matched } & \multirow[b]{2}{*}{ ASD } & \multicolumn{2}{|c|}{ After Propensity Score Matched } & \multirow[b]{2}{*}{ ASD } \\
\hline & PSO $(\mathbf{N}=7833)$ & Non-PSO $(\mathbf{N}=31,332)$ & & PSO (N =7833) & Non-PSO ( $\mathbf{N}=7833)$ & \\
\hline Age & & & 0.000 & & & 0.000 \\
\hline$<40$ & $4539(57.9)$ & $18,156(57.9)$ & & $4539(57.9)$ & $4539(57.9)$ & \\
\hline $40-64$ & $2326(29.7)$ & $9304(29.7)$ & & $2326(29.7)$ & $2315(29.6)$ & \\
\hline$\geq 65$ & $968(12.4)$ & $3872(12.4)$ & & $968(12.4)$ & $979(12.5)$ & \\
\hline Mean \pm SD & $37.8 \pm 20.1$ & $37.8 \pm 20.1$ & 0.000 & $37.8 \pm 20.1$ & $37.9 \pm 20.3$ & 0.006 \\
\hline Sex & & & 0.000 & & & 0.004 \\
\hline Female & $3757(48.0)$ & I5,028 (48.0) & & $3757(48.0)$ & $3774(48.2)$ & \\
\hline Male & $4076(52.0)$ & $16,304(52.0)$ & & $4076(52.0)$ & $4059(51.8)$ & \\
\hline Urbanization & & & 0.000 & & & 0.000 \\
\hline Urban & $4783(6 I . I)$ & $19,070(60.9)$ & & $4783(61.1)$ & $479 \mid(6 I .2)$ & \\
\hline Suburban & $2416(30.8)$ & $9680(30.9)$ & & $2416(30.8)$ & 2421 (30.9) & \\
\hline Rural & $634(8.1)$ & $2582(8.2)$ & & $634(8.1)$ & $621(7.9)$ & \\
\hline Hypertension & $952(12.2)$ & $3133(10.0)$ & 0.069 & $952(12.2)$ & $951(12.1)$ & 0.000 \\
\hline Hyperlipidemia & $340(4.3)$ & $1078(3.4)$ & 0.047 & $340(4.3)$ & $320(4.1)$ & 0.013 \\
\hline Chronic liver disease & $407(5.2)$ & $1080(3.4)$ & 0.086 & $407(5.2)$ & $413(5.3)$ & 0.003 \\
\hline Chronic kidney disease & $52(0.7)$ & $140(0.4)$ & 0.029 & $52(0.7)$ & $38(0.5)$ & 0.024 \\
\hline Diabetes & $497(6.3)$ & $1373(4.4)$ & 0.087 & $497(6.3)$ & $483(6.2)$ & 0.007 \\
\hline COPD & $292(3.7)$ & $844(2.7)$ & 0.059 & $292(3.7)$ & $298(3.8)$ & 0.004 \\
\hline Autoimmune disease & $115(1.5)$ & $225(0.7)$ & 0.072 & $115(1.5)$ & $116(1.5)$ & 0.001 \\
\hline NSAIDs & $5301(67.7)$ & $18,056(57.6)$ & 0.209 & $530 I(67.7)$ & $5311(67.8)$ & 0.003 \\
\hline
\end{tabular}

Abbreviations: COPD, chronic obstructive pulmonary disease; NSAIDs, non-steroidal anti-inflammatory drugs; ASD, absolute standardized difference.

Table 2 Incidence Density of Gout Among Different Group

\begin{tabular}{|l|l|l|}
\hline & Non-PSO & PSO \\
\hline N & 7833 & 7833 \\
Person-years & 64,662 & 62,202 \\
No. of gout & 329 & 433 \\
ID (95\% C.I.) & $5.09(4.57-5.67)$ & $6.96(6.34-7.65)$ \\
Relative risk (95\% C.I.) & 1.0 (reference) & $1.37(1.19-1.58)$ \\
\hline
\end{tabular}

Abbreviation: ID, incidence density (per 1000 person-years).

Furthermore, after adjusting for sex, age, urbanization, comorbidities, and medication use, patients with psoriasis had a 1.38 times (95\% CI, 1.20-1.60) higher risk of developing gout than those without psoriasis (Table 3). The other risk factors for gout were older age, male sex, hypertension, and COPD. Older patients, particularly those aged $>65$ years exhibited a 3.6 times (95\% CI, 2.83-4.56) higher risk of developing gout than those aged $<40$ years. Male patients also demonstrated a risk of developing gout that was 3.15 times (95\% CI, 2.63-3.78) higher than that in female patients. Furthermore, a higher risk of developing gout was observed among patients with various comorbidities, including hypertension (adjusted HR [aHR], 1.86; 95\% CI, 1.52-2.27) and COPD (aHR, 1.36; 95\% CI, 1.04-1.79). Patients taking NSAIDs (aHR, 0.39; 95\% CI, 0.34-0.45) had a decreased risk of developing gout.

Table 4 depicts the incidence and HR for gout among the subgroups stratified by age, sex, urbanization, comorbidities, and NSAID use. When stratified by age, patients aged $<65$ years and $\geq 65$ years in the psoriasis cohort had a higher HR than those belonging to the corresponding age groups in the non-psoriasis cohort. When stratified by sex, the men in the psoriasis cohort had a significantly higher HR for gout than those in the non-psoriasis cohort (HR, 1.43 [95\% CI, $1.22-1.68]$ ). The psoriasis cohort comprising women had an aHR of 1.24 (95\% CI, 0.90-1.71). When stratified by NSAID use, the patients of the psoriasis cohort who did not use NSAIDs showed a significantly higher risk of developing gout (aHR, 1.65; 95\% CI, 1.33-2.05) than those who did (aHR, 1.21; 95\% CI, 1.00-1.47). Supplementary Figure S2 
Table 3 Estimation the Hazard Ratio of Gout by Using Cox Proportional Hazard Regression

\begin{tabular}{|c|c|c|}
\hline & Crude HRs & Adjusted HRs ${ }^{a}$ \\
\hline & HR (95\% C.I.) & HR (95\% C.I.) \\
\hline \multicolumn{3}{|l|}{ Group } \\
\hline Non-PSO & I.0 (reference) & I.0 (reference) \\
\hline PSO & $1.36(1.18-1.57)$ & $1.38(1.20-1.60)$ \\
\hline \multicolumn{3}{|l|}{ Age } \\
\hline$<40$ & I.0 (reference) & I.0 (reference) \\
\hline $40-64$ & $3.64(3.07-4.32)$ & $3.04(2.55-3.63)$ \\
\hline$\geq 65$ & $5.53(4.55-6.73)$ & $3.60(2.83-4.56)$ \\
\hline \multicolumn{3}{|l|}{ Sex } \\
\hline Female & I.0 (reference) & I.0 (reference) \\
\hline Male & $3.97(3.32-4.74)$ & $3.15(2.63-3.78)$ \\
\hline \multicolumn{3}{|l|}{ Urbanization } \\
\hline Urban & I.0 (reference) & I.0 (reference) \\
\hline Suburban & $1.05(0.90-1.23)$ & $1.03(0.88-1.20)$ \\
\hline Rural & $1.19(0.93-1.53)$ & $1.08(0.84-1.40)$ \\
\hline Hypertension & $3.40(2.90-4.00)$ & $1.86(1.52-2.27)$ \\
\hline Hyperlipidemia & $2.53(1.95-3.28)$ & I.28 (0.97-I.69) \\
\hline Chronic liver disease & $\mathrm{I} .85(\mathrm{I} .43-2.39)$ & $\mathrm{I} .05(0.8 \mathrm{I}-\mathrm{I} .37)$ \\
\hline Chronic kidney disease & $2.48(1.18-5.23)$ & I. $17(0.55-2.49)$ \\
\hline Diabetes & $2.15(1.70-2.72)$ & $0.83(0.65-1.08)$ \\
\hline COPD & $3.12(2.43-4.01)$ & $1.36(1.04-1.79)$ \\
\hline Autoimmune disease & $1.37(0.8 \mathrm{I}-2.32)$ & $1.15(0.67-1.96)$ \\
\hline NSAIDs & $0.43(0.37-0.50)$ & $0.39(0.34-0.45)$ \\
\hline
\end{tabular}

Note: ${ }^{a}$ Adjusted for age, sex, urbanization, hypertension, hyperlipidemia, chronic liver disease, chronic kidney disease, diabetes, COPD, autoimmune disease, and NSAIDs.

Abbreviations: COPD, chronic obstructive pulmonary disease; NSAID, non-steroidal antiinflammatory drugs.

shows Kaplan-Meier curves stratified by NSAID use and demonstrated that patients with psoriasis who used NSAIDs were at a lower risk of developing gout than those who did not.

Table 5 depicts the comparison of aHRs of gout between the psoriasis and non-psoriasis cohorts using sensitivity analysis. The results showed that the psoriasis cohort exhibited a notably higher risk of developing gout (aHR, 1.96; 95\% CI, 1.33-2.88) than the non-psoriasis cohort. According to the results of the sensitivity analysis, the HR estimate of the psoriasis effect was also higher.

\section{Discussion}

Our study provides empirical evidence for the association between psoriasis and gout in a large nationwide sample. First, we found that patients with psoriasis exhibited a 1.3-fold increased risk of developing gout compared with those without psoriasis. Second, male sex, age $\geq 65$ years, hypertension, and COPD were significantly associated with the development of gout. Furthermore, we noted a negative association between gout and oral NSAID use in patients with psoriasis compared with that in patients without psoriasis.

In this nationwide, population-based study using data from the NHIRD of Taiwan, we found that the prevalence of gout in patients with psoriasis was higher than that in those without psoriasis. This finding was consistent with that of a previous cohort study in the United States, which showed that patients with psoriasis exhibit a 1.71 times higher risk of developing gout. ${ }^{13}$ The prevalence of gout is one among the highest in Taiwan (6.2\%), while it is $3.9 \%$ in the United States. ${ }^{13,21}$ Although ethnicity strongly influences gout prevalence, large population cohort studies in both Taiwan and the United States have suggested a significant association between psoriasis and gout. ${ }^{21}$ This may be because patients with psoriasis have increased levels of Th1 and Th17 cytokines in the skin lesions, joints, and 
Table 4 Subgroup Analysis of the Association Between Psoriasis and Gout

\begin{tabular}{|c|c|c|c|}
\hline & PSO & Non-PSO & HR (95\% C.I.) \\
\hline & No. of gout (\%) & No. of gout (\%) & \\
\hline $\mathrm{Age}^{\mathrm{a}}$ & & & \\
\hline$<65$ & $133(2.9)$ & $78(1.7)$ & $1.40(1.19-1.66)$ \\
\hline$\geq 65$ & $108(11.2)$ & $83(8.5)$ & $1.37(1.03-1.82)$ \\
\hline $\operatorname{Sex}^{\mathrm{a}}$ & & & \\
\hline Female & $82(2.2)$ & $68(1.8)$ & I.24 (0.90-I.7I) \\
\hline Male & $35 I(8.6)$ & $26 I(6.4)$ & $1.43(1.22-1.68)$ \\
\hline Urbanization & & & \\
\hline Urban & $267(5.6)$ & $186(3.9)$ & $1.53(1.27-1.85)$ \\
\hline Suburban & $|2|(5.0)$ & II 8 (4.9) & I.II (0.86-I.43) \\
\hline Rural & $45(7.1)$ & $25(4.0)$ & $1.88(1.15-3.06)$ \\
\hline Hypertensio & & & \\
\hline No & $319(4.6)$ & $236(3.4)$ & $1.44(1.22-1.70)$ \\
\hline Yes & $114(12.0)$ & $93(9.8)$ & $1.26(0.96-1.66)$ \\
\hline Hyperlipiden & & & \\
\hline No & $396(5.3)$ & $304(4.0)$ & $1.40(1.20-1.62)$ \\
\hline Yes & $37(10.9)$ & $25(7.8)$ & $1.43(0.85-2.39)$ \\
\hline Chronic live & & & \\
\hline No & $398(5.4)$ & $300(4.0)$ & $1.42(1.22-1.65)$ \\
\hline Yes & $35(8.6)$ & $29(7.0)$ & $1.33(0.81-2.18)$ \\
\hline Chronic kidr & & & \\
\hline No & $427(5.5)$ & $328(4.2)$ & $1.39(1.20-1.61)$ \\
\hline Yes & $6(11.5)$ & I (2.6) & $4.45(0.34-58.54)$ \\
\hline Diabetes $^{\mathrm{a}}$ & & & \\
\hline No & $386(5.3)$ & $297(4.0)$ & $1.39(1.19-1.61)$ \\
\hline Yes & $47(9.5)$ & $32(6.6)$ & $1.47(0.94-2.32)$ \\
\hline COPD $^{a}$ & & & \\
\hline No & $398(5.3)$ & 296 (3.9) & $1.43(1.23-1.67)$ \\
\hline Yes & $35(12.0)$ & $33(11.1)$ & 1.09 (0.67-I.77) \\
\hline Autoimmun & & & \\
\hline No & $423(5.5)$ & $325(4.2)$ & $1.38(1.20-1.60)$ \\
\hline Yes & $10(8.7)$ & $4(3.4)$ & $2.87(0.85-9.62)$ \\
\hline NSAIDs $^{\mathrm{a}}$ & & & \\
\hline No & $202(8.0)$ & $134(5.3)$ & $1.65(1.33-2.05)$ \\
\hline Yes & $231(4.4)$ & 195 (3.7) & 1.21 (1.00-I.47) \\
\hline
\end{tabular}

Notes: ${ }^{a}$ Adjusted for all variables; ${ }^{b}$ Adjusted for all variables, excluding chronic kidney disease and autoimmune disease; ${ }^{\mathrm{c} A d j u s t e d}$ for all variables, excluding urbanization and autoimmune disease; ${ }^{\mathrm{d}}$ Adjusted for all variables, excluding urbanization. Abbreviations: COPD, chronic obstructive pulmonary disease; NSAIDs, non-steroidal anti-inflammatory drugs.

Table 5 Sensitivity Analysis in the Estimation of the Gout Risk for PSO Exposure in Age-Matched and Sex-Matched Population

\begin{tabular}{|l|l|l|}
\hline & Univariate & Multivariate $^{\mathbf{a}}$ \\
\cline { 2 - 3 } & HR (95\% C.I.) & aHR (95\% C.I.) \\
\hline Group & & \\
Non-PSO & 1.0 (reference) & 1.0 (reference) \\
PSO & $1.89(1.29-2.77)$ & $1.96(1.33-2.88)$ \\
\hline
\end{tabular}

Note: ${ }^{2}$ Adjusted for age, sex, urbanization, hypertension, hyperlipidemia, chronic liver disease, chronic kidney disease, diabetes, COPD, and NSAIDs. 
blood. ${ }^{22,23}$ The Th1 and Th17 cytokine pathways play an important role in the pathogenesis of gout, and patients with gout have elevated levels of Th1 and Th17 cytokines in the peripheral blood and joints. ${ }^{24-26}$ The presence of common cytokine pathways may underlie the association between psoriasis and gout. In addition, elevated serum uric acid levels, ${ }^{27-29}$ which may be a result of increased keratinocyte renewal in patients with psoriasis, lead to the increased production of purine metabolites. ${ }^{30}$ These studies point to possible statistical differences between psoriasis and gout.

Additionally, after adjusting for covariates, this study revealed that hypertensive individuals were 1.86 times more likely to develop gout; this was consistent with the result of a systematic review and meta-analysis of cohort studies, which suggested that hypertension is an independent risk factor for gout. ${ }^{31}$ The clinical implication of these associations is significant because studies have indicated that hyperuricemia and hypertension severity may increase the risk of ischemic stroke. ${ }^{32}$ Diuretics used to treat hypertension may affect serum uric acid levels or induce flares and make gout treatment challenging. ${ }^{33}$ Meanwhile, the antihypertensive medication, losartan may help treat gout by reducing blood uric acid levels. ${ }^{9}$ Therefore, clinicians should be aware that patients with these risk factors are highly likely to develop gout and provide them the appropriate treatment.

An unanticipated finding of this study was the significant association between COPD and gout. This may be related to hyperuricemia in secondary polycythemia due to COPD. ${ }^{34}$ Hypoxia increases the metabolism of nucleotides, thereby increasing the generation of purines that are metabolized to uric acid, which can lead to hyperuricemia. $^{35,36}$

Another important finding of this study was that age, sex, and NSAID use were significantly associated with the incidence of gout. In particular, the strongest association was observed in men and those aged $>65$ years, which is consistent with the findings of previous studies. Although psoriasis mostly occurs at age $<40$ years, our study results indicate that gout may occur at any age, particularly in men. NSAID use was also associated with a decreased risk of gout. We also found that the interaction between NSAID use and psoriasis had an impact on gout risk. Our study results reveal that NSAID use is an important protective as well as modifying factor for gout incidence. NSAIDs can modify and repair the negative effects of psoriasis on gout and are the most common drugs used to treat acute gout attacks, unless they are contraindicated for the patient. To the best of our knowledge, this is the first cohort study to demonstrate this finding. However, the underlying mechanisms have not yet been clearly established. A previous study reported that NSAID use may substantially increase urinary uric acid excretion. ${ }^{33}$ Further randomized, placebo-controlled trials are warranted to assess the benefits of NSAIDs for gout.

This study has several strengths. First, we analyzed a large population-based cohort to determine the association between psoriasis and gout. Second, all hospital and clinic visits were recorded in the NHIRD, thereby reducing the possibility of referral bias. Third, the HRs were calculated based on the initially selected groups and adjusted using multiple Cox regression and sensitivity analyses. All HRs obtained in the models used indicated that patients with psoriasis had a higher risk of developing gout than those without psoriasis. These results provide further evidence that the increased risk of gout in patients with psoriasis is not affected or modified by other comorbidities.

This study has some limitations. First, the NHIRD does not contain information on disease severity or laboratory test results. According to the sensitivity analysis, inclusion of dermatologists and rheumatologists in the study indicated that the severity of the diagnosed disease was higher. Therefore, the strength of the association based on disease severity may be determined. Second, diagnostic coding in a medical claims database may not be accurate. However, most diagnoses of psoriasis and gout were made by dermatologists or rheumatologists and the sensitivity analysis we conducted to enhance accuracy ensured that misdiagnosis did not affect the study results.

\section{Conclusion}

In summary, our findings demonstrate that gout is significantly associated with psoriasis, and the strength of the association increases with risk factors, such as male sex, older age, hypertension, and COPD. Therefore, increased clinical vigilance is required to prevent the possible coexistence of gout and psoriasis. However, the biological mechanisms underlying this association remain unclear. Further research is necessary to elucidate the molecular mechanisms involved in this process. 


\section{Acknowledgments}

The authors thank all patients and staff who made this study possible. James Cheng-Chung Wei and Yi-Jung Chang are co-first authors for this study.

\section{Funding}

There is no funding to report.

\section{Disclosure}

The authors report no conflicts of interest in this work.

\section{References}

1. Christophers E. Psoriasis-epidemiology and clinical spectrum. Clin Exp Dermatol. 2001;26(4):314-320. doi:10.1046/j.1365-2230.2001.00832.x

2. Augustin M, Radtke MA. Quality of life in psoriasis patients. Expert Rev Pharmacoecon Outcomes Res. 2014;14(4):559-568. doi:10.1586/ 14737167.2014.914437

3. Javitz HS, Ward MM, Farber E, et al. The direct cost of care for psoriasis and psoriatic arthritis in the United States. J Am Acad Dermatol. 2002;46 (6):850-860. doi:10.1067/mjd.2002.119669

4. Vanderpuye-Orgle J, Zhao Y, Lu J, et al. Evaluating the economic burden of psoriasis in the United States. J Am Acad Dermatol. 2015;72(6):961967.e5. doi:10.1016/j.jaad.2015.02.1099

5. Parisi R, Iskandar IYK, Kontopantelis E, et al. National, regional, and worldwide epidemiology of psoriasis: systematic analysis and modelling study. BMJ. 2020;369:m1590. doi:10.1136/bmj.m1590

6. Rendon A, Schäkel K. Psoriasis pathogenesis and treatment. Int J Mol Sci. 2019;20(6):1475. doi:10.3390/ijms20061475

7. Armstrong AW, Read C. Pathophysiology, clinical presentation, and treatment of psoriasis: a review. JAMA. 2020;323(19):1945-1960. doi:10.1001/ jama.2020.4006

8. Springate DA, Parisi R, Kontopantelis E, et al. Incidence, prevalence and mortality of patients with psoriasis: a U.K. population-based cohort study. Br J Dermatol. 2017;176(3):650-658. doi:10.1111/bjd.15021

9. Singh JA, Gaffo A. Gout epidemiology and comorbidities. Semin Arthritis Rheum. 2020;50(3S):S11-S16. doi:10.1016/j.semarthrit.2020.04.008

10. Yu KH, Chen DY, Chen JH, et al. Management of gout and hyperuricemia: multidisciplinary consensus in Taiwan. Int J Rheum Dis. 2018;21 (4):772-787. doi:10.1111/1756-185X.13266

11. Dehlin M, Jacobsson L, Roddy E. Global epidemiology of gout: prevalence, incidence, treatment patterns and risk factors. Nat Rev Rheumatol. 2020;16(7):380-390. doi:10.1038/s41584-020-0441-1

12. Bardin T, Richette P. Impact of comorbidities on gout and hyperuricaemia: an update on prevalence and treatment options. BMC Med. 2017;15 (1):123. doi:10.1186/s12916-017-0890-9

13. Merola JF, Wu S, Han J, et al. Psoriasis, psoriatic arthritis and risk of gout in US men and women. Ann Rheum Dis. 2015;74(8):1495-1500. doi:10.1136/annrheumdis-2014-205212

14. Hu SC, Lin CL, Tu HP. Association between psoriasis, psoriatic arthritis and gout: a nationwide population-based study. $J$ Eur Acad Dermatol Venereol. 2019;33(3):560-567. doi:10.1111/jdv.15290

15. Chen Z, Xu Y, Chen M, et al. Gout augments the risk of cardiovascular disease in patients with psoriasis: a population-based cohort study. Front Immunol. 2021;12:703119. doi:10.3389/fimmu.2021.703119

16. Caso F, Del Puente A, Oliviero F, et al. Metabolic syndrome in psoriatic arthritis: the interplay with cutaneous involvement. Evidences from literature and a recent cross-sectional study. Clin Rheumatol. 2018;37(3):579-586. doi:10.1007/s10067-017-3975-0

17. Chen TT, Yeh YC, Chien KL, et al. Risk of infective endocarditis after invasive dental treatments: case-only study. Circulation. 2018;138 (4):356-363. doi:10.1161/CIRCULATIONAHA.117.033131

18. Wu CY, Kuo KN, Wu MS, et al. Early Helicobacter pylori eradication decreases risk of gastric cancer in patients with peptic ulcer disease. Gastroenterology. 2009;137(5):1641-1648.e1-2. doi:10.1053/j.gastro.2009.07.060

19. Chen ML, Kao WM, Huang JY, et al. Human papillomavirus infection associated with increased risk of new-onset psoriasis: a nationwide population-based cohort study. Int J Epidemiol. 2020;49(3):786-797. doi:10.1093/ije/dyaa027

20. Austin PC. Balance diagnostics for comparing the distribution of baseline covariates between treatment groups in propensity-score matched samples. Stat Med. 2009;28(25):3083-3107. doi:10.1002/sim.3697

21. Kuo CF, Grainge MJ, See LC, et al. Epidemiology and management of gout in Taiwan: a nationwide population study. Arthritis Res Ther. 2015;17 (1):13. doi:10.1186/s13075-015-0522-8

22. Ogawa E, Sato Y, Minagawa A, et al. Pathogenesis of psoriasis and development of treatment. J Dermatol. 2018;45(3):264-272. doi:10.1111/13468138.14139

23. Diani M, Altomare G, Reali E. T helper cell subsets in clinical manifestations of psoriasis. J Immunol Res. 2016;2016:7692024. doi:10.1155/2016/ 7692024

24. Luo G, Yi T, Zhang G, et al. Increased circulating Th22 cells in patients with acute gouty arthritis: a CONSORT-compliant article. Medicine. 2017;96(42):e8329. doi:10.1097/MD.0000000000008329

25. Liu Y, Zhao Q, Yin Y, et al. Serum levels of IL17 are elevated in patients with acute gouty arthritis. Biochem Biophys Res Comm. 2018;497 (3):897-902. doi:10.1016/j.bbrc.2018.02.166

26. Scanu A, Oliviero F, Ramonda R, et al. Cytokine levels in human synovial fluid during the different stages of acute gout: role of transforming growth factor beta1 in the resolution phase. Ann Rheum Dis. 2012;71(4):621-624. doi:10.1136/annrheumdis-2011-200711 
27. Kwon HH, Kwon IH, Choi JW, et al. Cross-sectional study on the correlation of serum uric acid with disease severity in Korean patients with psoriasis. Clin Exp Dermatol. 2011;36(5):473-478. doi:10.1111/j.1365-2230.2010.03988.x

28. Jain VK, Lal H. C-reactive protein and uric acid levels in patients with psoriasis. Indian J Clin Biochem. 2011;26(3):309-311. doi:10.1007/s12291011-0132-4

29. Gisondi P, Targher G, Cagalli A, et al. Hyperuricemia in patients with chronic plaque psoriasis. J Am Acad Dermatol. 2014;70(1):127-130. doi:10.1016/j.jaad.2013.09.005

30. Choi HK, Atkinson K, Karlson EW, et al. Purine-rich foods, dairy and protein intake, and the risk of gout in men. $N$ Engl $J$ Med. 2004;350 (11):1093-1103. doi:10.1056/NEJMoa035700

31. Evans PL, Prior JA, Belcher J, et al. Obesity, hypertension and diuretic use as risk factors for incident gout: a systematic review and meta-analysis of cohort studies. Arthritis Res Ther. 2018;20(1):136. doi:10.1186/s13075-018-1612-1

32. Chaudhary NS, Bridges SL, Saag KG, et al. Severity of hypertension mediates the association of hyperuricemia with stroke in the REGARDS case cohort study. Hypertension. 2020;75(1):246-256. doi:10.1161/HYPERTENSIONAHA.119.13580

33. Fatima T, Nilsson PM, Turesson C, et al. The absolute risk of gout by clusters of gout-associated comorbidities and lifestyle factors-30 years follow-up of the Malmö preventive project. Arthritis Res Ther. 2020;22(1):244. doi:10.1186/s13075-020-02339-0

34. Kohkhar N. Hyperuricemia and gout in secondary polycythemia due to chronic obstructive pulmonary disease. $J$ Rheumatol. 1980;7(1):114-116.

35. Hasday JD, Grum CM. Nocturnal increase of urinary uric acid: creatinine ratio. A biochemical correlate of sleep-associated hypoxemia. Am Rev Respir Dis. 1987;135(3):534-538. doi:10.1164/arrd.1987.135.3.534

36. Glantzounis GK, Tsimoyiannis EC, Kappas AM, et al. Uric acid and oxidative stress. Curr Pharm Des. 2005;11(32):4145-4151. doi:10.2174/ 138161205774913255

Clinical Epidemiology

Dovepress

\section{Publish your work in this journal}

Clinical Epidemiology is an international, peer-reviewed, open access, online journal focusing on disease and drug epidemiology, identification of risk factors and screening procedures to develop optimal preventative initiatives and programs. Specific topics include: diagnosis, prognosis, treatment, screening, prevention, risk factor modification, systematic reviews, risk \& safety of medical interventions, epidemiology \& biostatistical methods, and evaluation of guidelines, translational medicine, health policies \& economic evaluations. The manuscript management system is completely online and includes a very quick and fair peer-review system, which is all easy to use.

Submit your manuscript here: https://www.dovepress.com/clinical-epidemiology-journal 